\title{
EDITORIAL BOARD MEMBERS
}

\section{EDITOR-IN-CHIEF}

Kapil Amgain, MD(AM), MSc (Anatomy), Executive Director (Research Wings); Nirvana Psychosocial Counseling Centre and Research Institute; Nepal

\section{ASSOCIATE EDITOR}

Sunil Shrestha, Pharm. D. (Clinical Pharmacy); Nepal Cancer Hospital and Research Center, Nepal

\section{NATIONAL EDITORIAL BOARD MEMBERS}

Ganesh Amgain, MA (Psychology); Director (Founder), Nirvana Psychosocial Counseling Centre and Research Institute; Nepal

Navindra Raj Bista; MD, Department of Anesthesiology, Maharajgunj Medical Campus, Institute of Medicine, Tribhuvan University, Nepal

Kamal Raj Sharma, MD (Pediatrics); Professor of Department of Pediatrics; Karnali Academy of Health Sciences (KAHS); Nepal

Mandira Shahi, MHPE, Ph.D. Scholar, Professor \& Executive Director of National Centre of Health Professions Education (NCHPE), Institute of Medicine, Tribhuvan University; Nepal

Damaru Prasad Paneru; MPH, PhD; Associate Professor, Director of School of Health and Allied Sciences, Pokhara University, Nepal

Rajib Chaulagain, MDS, Ph.D. (Oral Biology); Assistant Professor, Department of Oral Pathology, Chitwan Medical College, Affiliated to Tribhuvan University, Nepal

Dillee P. Paudel, MPH, PhD; School of Health and Allied Sciences, Purbanchal University, Nepal

\section{INTERNATIONAL EDITORIAL}

\section{BOARD MEMBERS}

Mary Putera, LMHC, MDiv, CAGS, Ph.D. Scholar; United States of America (USA)

Arash Ziapour, MS, PhD, Iran University of Medical Sciences, Iran

Gakul Bhatta, MS (Surgery); United Kingdom (UK) Taraman Kadayat, M. Pharm, Ph.D., Research Scientist, Drug Discovery Chemistry Group, New Drug Development Center, DGMIF, South Korea
Ahmad Naeem Sajed, M.Sc., M.Phil. (Microbiology) Ph.D. Scholar, Department of Microbiology, The Lahore Garrison University, Lahore, Pakistan

Ashok Silwal, MS (Pharmaceutical sciences), Southern Illinois University; Edwardsville; USA

Tulsi C. Singel, MS (Anatomy); Professor and Head of Department of Anatomy, Guru Govinda University, Zydus medical college $\mathrm{n}$ hospital; India

Sherine Adel Nasry, MDS, Ph.D.; Professor of Department of Oral and Dental Research Division, National Research Centre; Egypt

Sandra Maria Martin, Midwifery (Nursing); Ystad/ Malmö Labour-Ward Malmö University Hospital/ Ystad Hospital, Sweden

S. M. Kadri, MPH/ICHD, Directorate of Health Services (DHS) Kashmir, India

Mohit Zamad, MDS (Oral \& Maxillofacial Surgery), Department of Oral \& Maxillofacial Surgery, Chhatisgarh Dental College \& Research Centre, Rajnandgaon; India

Deepak Sharma, DVM, MSc (Physiology), Ph.D. Scholar (Neurophysiology); University of New South Wales; Australia

Nimmi Singh, MDS (Oral Medicine \& Radiology), Pioneer in CBCT; Indira Gandhi Institute of Medical Sciences and Hospital, Patna; India

Aarajana Shrestha, PhD, Yeungnam University, South Korea

Arshi Taj, MD; Government Medical College, Department of Anaesthesia critical care and Pain Management, Jammu and Kashmir, India

\section{STATISTICAL EDITOR}

Prem Prasad Panta, M.Sc. (Biostatistics); Associate Professor, School of Medicine, Karnali Academy of Health Sciences (KAHS); Nepal

\section{ASSISTANT EDITORS}

Amrita Chaulagain; Research Officer, Jeebanta Advanced Kathmandu Imaging, Kathmandu, Nepal

\section{TECHNICAL EDITOR}

Suresh Risal, Executive Director and Founder, Potent Media Home, Kathmandu, Nepal 